\title{
Covid-19: Prevalencia de Emociones Negativas y Malestar Psicológico en una Muestra de Adultos de la Ciudad de México
}

\author{
Covid-19: Prevalence of Negative Emotions and Psychological \\ Discomfort in a Sample of Adults from Mexico City
}

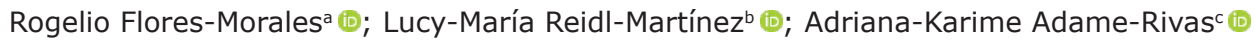

Recibido: 29-11-2020; Aceptado: 10-01-2021; Publicado: 25-01-2021.

\section{RESUMEN}

Objetivo: El presente estudio tuvo como objetivo identificar la prevalencia de emociones negativas y malestar psicológico en personas adultas de la Ciudad de México -la región más afectada del país por el virus SARS-CoV-2 (COVID-19)-, y analizar si existen diferencias según el género, la edad y la escolaridad. El levantamiento de datos se realizó a finales de la Fase 1 y al inicio de la Fase 2 de la pandemia. Método: La metodología empleada en esta investigación es cuantitativa y el diseño exploratorio, descriptivo y transversal. Se utilizó una muestra no representativa por invitación de 824 personas adultas de la Ciudad de México. Resultados: Los resultados globales muestran una prevalencia de $31.6 \%$ de malestar psicológico y una alta presencia de emociones negativas, siendo la incertidumbre (58.6\%), la preocupación (51.7\%), el fastidio $(49.8 \%)$ y la impotencia $(47.1 \%)$ las más presentes. Las mujeres y las personas jóvenes obtuvieron indicadores significativamente más elevados en todas las variables analizadas. También se identificó que la escolaridad no representó un factor de protección o de regulación con respecto a la presencia de emociones negativas y de malestar psicológico. Conclusiones: Los resultados corroboran, en términos cuantitativos, el fuerte impacto psicoemocional del Covid-19 en un contexto urbano latinoamericano, y enfatizan la necesidad de fortalecer políticas públicas que atiendan esta problemática psicosocial y sanitaria.

Palabras clave: emociones negativas; malestar psicológico; pandemias; Covid-19; SARS-CoV-2.

\section{ABSTRACT}

Objective: The present study aimed to identify the prevalence of negative emotions and psychological distress in adults in Mexico City - the region most affected by the SARS-CoV-2 virus (COVID-19) in the country-, and analyze whether there are differences according to gender, age

aUniversidad Nacional Autónoma de México (UNAM), Facultad de Psicología, Ciudad de México. rogelio.flores@psicologia.unam.mx bUniversidad Nacional Autónoma de México (UNAM), Facultad de Psicología, Ciudad de México. lucym@unam.mx

cUniversidad Nacional Autónoma de México (UNAM), Facultad de Psicología, Ciudad de México. akar_94@hotmail.com

Flores-Morales, R., Reidl-Martínez, L.M., Adame-Rivas, A.K. (2021). Covid-19: Prevalencia de Emociones Negativas y Malestar Psicológico en una Muestra de Adultos de la Ciudad de México. Búsqueda, v. 8, n. 1, e530. https://doi.org/10.21892/01239813.530 
and education. The data collection was carried out at the end of Phase 1 and at the beginning of Phase 2 of the pandemic. Method: The methodology used in this research is quantitative and the design is exploratory, descriptive and cross-sectional. A non-representative sample by invitation of 824 adults from Mexico City was used. Results: The global results show a prevalence of $31.6 \%$ of psychological discomfort and a high presence of negative emotions, being uncertainty $(58.6 \%)$, worry $(51.7 \%)$, annoyance $(49.8 \%)$ and impotence $(47.1 \%)$ the most present. Women and young people obtained significantly higher indicators in all the variables analyzed. It was also identified that schooling did not represent a protective or regulatory factor with respect to the presence of negative emotions and psychological distress. Conclusions: The results corroborate, in quantitative terms, the strong psycho-emotional impact of Covid-19 in a Latin American urban context and emphasize the need to strengthen public policies that address this psychosocial and health problem.

Keywords: negative emotions; psychological discomfort; pandemics; Covid-19; SARS-CoV-2.

\section{INTRODUCCIÓN}

La pandemia por SARS-CoV-2 que la Organización Mundial de la Salud (OMS) identificó oficialmente a finales de diciembre de 2019, representó el inicio de una crisis sanitaria sin precedentes a nivel global. A noviembre de 2021, se habían registrado más de 249 millones de casos confirmados y más de cinco millones de fallecimientos en todo el mundo, desencadenando una caótica sinergia de dolor, sufrimiento y duelo (Flores-Morales, 2020; OMS, 2021).

Paralelamente a dichas secuelas físicas, los impactos psicológicos en la salud mental comenzaron a identificarse en distintas zonas geográficas del planeta, siendo la ansiedad, la depresión y el estrés postraumático las categorías clínicas más reconocidas y estudiadas (Cao, et al., 2020; Gamonal, et al. 2020; Gao, et al., 2020; Liu, et al., 2020; Parrado-González \& León-Jariego, 2020; Rodríguez, et al., 2021; Roy, et al., 2020; Sandín, et al., 2020; Wang, et al., 2020). En el caso particular de México, se identificó que $50 \%$ de personas de una muestra no representativa estaba ansiosa y $28 \%$ presentó síntomas depresivos (Priego-Parra et al., 2020). Con respecto al estrés postraumático, se identificó una prevalencia de 27.7\% (González-Ramírez et al., 2020).

En la presente investigación, sin embargo, nos enfocamos en mostrar otro aspecto concurrente de este vasto conjunto de respuestas psicológicas asociadas a la pandemia: la presencia de emociones negativas en el contexto del Covid-19, las cuales, en su conjunto, podrían estar relacionadas, de algún modo, con la configuración de malestar psicológico.

Las emociones son una dimensión psicológica vital en la comprensión del ser humano, ya que no pocas veces orientan el comportamiento cotidiano de las personas, particularmente en tiempos de crisis. Aceptando que todavía no existe una definición consensuada al interior de la Psicología, sí tenemos la posibilidad de coincidir en que las emociones son experiencias multidimensionales que podrían ser agrupadas en al menos cuatro grandes dimensiones: 1 . Fisiológico/Adaptativo; 2. Cognitivo/Subjetivo/Evaluativo; 3. Comportamental/Expresivo; y 4. Sociocultural/Interpretativo (Calhoun \& Solomon, 1984; Chóliz, 2005; Le Breton, 2013; Marina, 1999; Ortony et al., 1996; Piqueras, et al., 2009).

En un intento de clasificación básico y preliminar de las emociones en un contexto pandémico, consideramos que es posible distinguir, en primera instancia, entre aquellas emociones que perturban/desagradan y aquellas que generan placer/agrado. En este sentido, a las primeras se les denomina negativas - puesto que remiten a cierta alteración que rompe con un estado otrora identificado como estable - mientras que a las segundas se les reconoce como emociones positivas (Chóliz. 2005; Piqueras, 2009). 
Flores-Morales, et al., (2021, p. 7) señalan que las emociones negativas y perturbadoras son "aquellas experiencias subjetivas que modifican o alteran el orden o la quietud de una persona." Es decir, el carácter negativo y perturbador de dichas emociones surge como resultado de un periodo, experiencia o situación de inestabilidad autopercibido - con frecuencia un evento complicado de afrontar, traumático o que coloca en riesgo la integridad de los individuos-, el cual regularmente cambia o modifica el estado de ánimo en términos nocivos para la propia persona. Por su parte, Piqueras, et al (2009), señalan que las emociones negativas son experiencias desagradables, como el miedo, la ansiedad, el enojo, la tristeza o la depresión, en contraposición con las emociones positivas, las cuales aluden regularmente a una sensación de placer o agrado.

Con respecto al malestar psicológico ocurre algo similar. Desde su diseño conceptual mediante el Cuestionario General de Salud de Goldberg (1979), este constructo ha tenido diversas modificaciones a lo largo de las últimas cuatro décadas. En un principio, como señala García (1999), el CGS fue elaborado con la intención de identificar la severidad de disturbios psiquiátricos menores, es decir, la salud mental autopercibida. Posteriormente, en su modalidad de 12 reactivos, el CGS se transformó en un modelo unidimensional que aludía a dos concepciones antagónicas: malestar/bienestar psicológico — también denominado malestar/bienestar emocional.

En este sentido, el constructo malestar psicológico autopercibido incluye diversas dimensiones, como sintomatología ansiosa y depresiva básicas, funcionamiento social y afrontamiento. Como señalan Godoy-Izquierdo et al., (2002) y Villa et al., (2013), el malestar psicológico agrupa particularmente a dos tipos de problemas: la incapacidad para realizar actividades diarias normales o adaptativas, y la manifestación de fenómenos perturbadores y síntomas psicopatológicos básicos a nivel personal y social.

En el contexto de la pandemia de Covid-19, resulta importante identificar cuáles son las principales emociones negativas experimentadas por personas adultas de la Ciudad de México, y reconocer si estas son susceptibles de coadyuvar eventualmente en la configuración de algún tipo de malestar psicológico. Identificando particularmente a las emociones negativas más recurrentes en la ciudad más afectada por el virus SAR-CoV-2, se podría tener una radiografía o panorama general de cómo se ha sentido un sector de mexicanos en el contexto pandémico, en aras de intentar vislumbrar las posibles consecuencias futuras de esta nueva normalidad subjetiva.

Por lo anterior, en la presente investigación se plantean los siguientes objetivos:

1. Evaluar la prevalencia de emociones negativas y de malestar psicológico en una muestra no representativa de personas adultas de la Zona Metropolitana de la Ciudad de México (ZMCM) en el contexto pandémico del Covid-19.

2. Identificar si existen diferencias en ambas variables según el género, rango de edad y escolaridad de los participantes.

\section{MÉTODO}

Diseño de Investigación. En esta investigación se empleó una metodología cuantitativa con diseño exploratorio y descriptivo. Debido a que los datos aquí proporcionados se obtuvieron en un solo corte temporal, el presente estudio es de carácter transversal (Shaughnessy et al., 2007).

Participantes. En el presente estudio se empleó una muestra intencional por invitación en cadena por redes, también conocida como bola de nieve (Hernández, et al., 2014). Participaron 824 personas de la Zona Metropolitana de la Ciudad de México (ZMCM), de las cuales $77.8 \%$ fueron mujeres y $22.2 \%$ hombres. La edad mínima identificada fue 18 y la máxima $75(M=33.36)$. En esta investigación se clasificaron cuatro rangos de edad: Jóvenes de 18 a 24 años (35.9\% 
de la muestra); adultos jóvenes de 25 a 44 años (41.5\%); adultos maduros de 45 a 59 años $(17.8 \%)$; y adultos mayores de 60 años en adelante $(4.7 \%)$.

Con respecto a la escolaridad, $0.2 \%$ cursó únicamente educación primaria, $1.6 \%$ secundaria, 9.2\% Preparatoria (Educación Media Superior), 40.4\% eran estudiantes de licenciatura, 35.9\% tenían una licenciatura finalizada y $12.6 \%$ contaban con un posgrado terminado o en curso.

En un inicio se invitó a estudiantes de dos universidades de la Ciudad de México para que participaran en el estudio. Una vez que respondieron los instrumentos, se les pidió que compartieran el formulario con familiares y amigos con el fin de ampliar el espectro del estudio. De esta forma, se pudo alcanzar una muestra final de 824 participantes.

Los criterios de inclusión para este estudio fueron los siguientes: 1) Tener 18 años o más; y 2) Vivir en la Zona Metropolitana de la Ciudad de México (ZMCM) al momento de la pandemia (puesto que ésta fue la región geográfica más afectada del país por el virus SARS-CoV-2). Los criterios de exclusión, en consecuencia, fueron los siguientes: 1) Ser menor de edad; y 2) Vivir en cualquier otro estado de la república que no fuera la ZMCM.

Instrumentos. IREN-35. Se aplicó el Inventario de Respuestas Emocionales Negativas y Perturbadoras en Contextos de Pandemias (IREN-35) (Flores-Morales, et al., 2021). El instrumento es válido y confiable para población mexicana, e identifica la presencia de emociones negativas relacionadas con la exposición directa o indirecta a una pandemia, incluida la experiencia de aislamiento físico y social que ésta provoca. El instrumento está constituido por cuatro factores, con un Alfa de Cronbach global de .96. Las dimensiones del inventario son las siguientes: 1) Emociones Negativas por Distanciamiento Físico y/o Social (ENDFS) $(a=.93)$; 2 ) Emociones Negativas por Pérdida/Ausencia de Estabilidad o Bienestar (ENPEB) $(a=.91)$; 3) Emociones Negativas por Percepción de Amenaza (ENPA) $(a=.88)$; y 4) Emociones Negativas por Percepción de Peligro Intenso (ENPPI) $(a=.90)$. El inventario se responde usando una escala Likert de cinco opciones: Nada presente (1), Poco presente (2), Más o menos presente (3), Muy presente (4) y Demasiado presente (5).

Cabe señalar que en este estudio se decidió incluir una emoción adicional que en el artículo original (Flores-Morales, et al., 2021) fue eliminada por el análisis factorial y que, sin embargo, consideramos está muy presente en contextos pandémicos: el miedo. Al realizar el análisis factorial en el presente estudio, el miedo se ubicó en la dimensión "Emociones por percepción de amenaza", con una carga factorial de .590. En este sentido, la versión del IREN aplicada en esta investigación estuvo constituida por 36 reactivos y no por 35 como se planteó en la versión original. Dicha inclusión no modificó ninguno de los factores reportados por Flores-Morales, et al. (2021).

CGS. También se aplicó la versión mexicana del Cuestionario General de Salud de Goldberg y Williams de 12 reactivos (CGS) (Solís-Cámara et al., 2016). El CGS evalúa la salud autopercibida, el estado de bienestar general, y particularmente la presencia de malestar psicológico (Goldberg \& Hillier, 1979; Goldberg \& Williams, 1988; Espíndola, 2006). La versión estandarizada de 12 reactivos para población mexicana está constituida por una dimensión (bienestar/malestar psicológico) y tiene un alfa total de .81. El CGS se presenta con una escala Likert en donde las opciones de respuesta van de 0 a 3 . De acuerdo con los autores, un puntaje igual o mayor a 17 representa deterioro en la salud mental o presencia de malestar psicológico.

Cuestionario Sociodemográfico. Adicionalmente se aplicó un conjunto de preguntas que indagan sobre el género, edad y escolaridad de los participantes. 
Procedimiento. El levantamiento de datos se realizó durante la Fase 2 y principios de la Fase 3 de Covid-19 (de marzo a junio de 2020). En dicho periodo, se registraron 226 mil personas contagiadas y 27 mil 769 defunciones por el virus SARS-Cov-2 en México (Secretaría de Salud, 2020). La declaratoria de la Fase 2 se instauró el 23 de marzo de 2020 y la Fase 3, el 21 de abril. En aquel momento las autoridades sanitarias informaron que tomaron dicha decisión al identificar que en cuatro regiones del país ya se habían superado los mil casos, particularmente en la zona centro de México, con más de cuatro mil personas contagiadas (casi la mitad de todo el país) (Redacción AN, 2020). Justamente ésta es la zona y el rango temporal que abarca el presente estudio.

Los instrumentos se aplicaron vía Internet, a través de la Plataforma de Formularios de Google, justamente para evitar el contacto físico con los informantes y garantizarles la seguridad en su participación.

Análisis de Datos. En primera instancia, con el fin de identificar si ambos instrumentos utilizados en esta investigación eran válidos y confiables, se realizó un análisis factorial exploratorio con rotación ortogonal varimax, bajo el método de extracción de máxima verosimilitud. También se examinó la confiabilidad total y por factores del IREN y el CGS mediante el índice Alfa de Cronbach.

Posteriormente se realizaron análisis descriptivos de todas las variables sociodemográficas: género, rango de edad y escolaridad. Para identificar las prevalencias de las dos variables principales (emociones negativas y malestar psicológico) se realizaron frecuencias para cada una de ellas. Asimismo, con el objetivo de identificar las diferencias entre las variables aludidas, se empleó la $U$. de Mann Whitney y Chi cuadrada. Todos los análisis estadísticos se realizaron con el programa SPSS versión 25.

Consideraciones Éticas. En la Plataforma de Google que contenía los instrumentos aplicados, se incluyó una carta de consentimiento informado en donde se les explicó a los participantes los objetivos y alcances de la investigación a la que se les estaba invitando. A los participantes se les garantizó estricta confidencialidad, añadiendo que, si en algún momento de la aplicación de los instrumentos decidían retirarse, estaban en su completo derecho de hacerlo. Una vez aprobado su consentimiento informado, se procedió con la recolección de datos. Algunos de los participantes solicitaron que se les enviara la investigación una vez ésta se hubiere publicado.

\section{RESULTADOS}

Para tener certeza de la validez y confiabilidad de los instrumentos empleados en este estudio, en primera instancia se realizó un análisis factorial que incluyó los 35 reactivos (emociones negativas) de la versión original del IREN, al que se le agregó una emoción adicional: el miedo. En el análisis realizado se obtuvo una estructura final de cuatro dimensiones que explicó en su conjunto el $57 \%$ de la varianza. Al realizar los análisis de confiabilidad, se encontró que el IREN obtuvo un valor a de Cronbach global de .96, mientras que el Cuestionario General de Salud (CGS) de .88. Este último estuvo constituido por una sola dimensión en coincidencia con la versión original.

Una vez corroborado que ambos instrumentos fueron válidos y confiables se procedió a identificar las prevalencias de cada una de las emociones negativas emergentes. Se encontró que la incertidumbre $(58.6 \%, n=483)$, la preocupación $(51.7 \%, n=426)$, el fastidio $(49.8 \%, n=336)$, la impotencia (47.1\%, $n=388)$, la impaciencia $(44.8 \% ; n=369)$, la flojera $(44.1 \%, n=363)$, la 
inquietud (41.7\%, $n=344)$, la vulnerabilidad (38.2\%, $n=315)$, el miedo $(37.5 \%, n=309)$ y la inseguridad $(35.8 \%, \mathrm{n}=295)$, fueron las 10 emociones más recurrentes en la muestra total. Por su parte, las emociones con menor presencia fueron el recelo $(9.7 \%, n=80)$, pavor $(10.3 \%$, $\mathrm{n}=85$ ) y desdicha $(10.4 \%, \mathrm{n}=86)$. En 22 de las 36 emociones del IREN se identificaron diferencias significativas, en donde las mujeres obtuvieron los puntajes más elevados (Tabla 1).

Tabla 1. Las 10 emociones negativas más frecuentes en el contexto del Covid-19 durante la Fase 1 y 2 de la pandemia en ZMCM.

\begin{tabular}{|c|c|c|c|c|c|}
\hline \multirow[b]{2}{*}{ Emoción } & \multicolumn{5}{|c|}{ Porcentaje de personas que experimentaron la emoción } \\
\hline & $\begin{array}{l}\text { Factor o } \\
\text { dimensión }\end{array}$ & $\begin{array}{l}\text { Muestra total } \\
(N=824)\end{array}$ & $\begin{array}{l}\text { Mujeres } \\
(n=641)\end{array}$ & $\begin{array}{l}\text { Hombres } \\
(n=183)\end{array}$ & $x^{2}$ \\
\hline 1. Incertidumbre & ENPA & 58.6 & 61 & 50.3 & $6.75 * *$ \\
\hline 2. Preocupación & ENPA & 51.7 & 55.2 & 39.3 & $14.38 * * *$ \\
\hline 3. Fastidio & ENDFS & 49.8 & 43.2 & 32.2 & $7.09 * *$ \\
\hline 4. Impotencia & ENPA & 47.1 & 51.6 & 31.1 & $23.99 * * *$ \\
\hline 5. Impaciencia & ENDFS & 44.8 & 47.9 & 33.9 & $11.30 * * *$ \\
\hline 6. Pereza / Flojera & ENDFS & 44.1 & 44.6 & 42.1 & 0.37 \\
\hline 7. Inquietud & ENPA & 41.7 & 45.6 & 28.4 & $17.19 * * *$ \\
\hline 8. Vulnerabilidad & ENPA & 38.2 & 40.4 & 30.6 & 5.79* \\
\hline 9. Miedo & ENPA & 37.5 & 41.8 & 22.4 & $22.87 * * *$ \\
\hline 10. Inseguridad & ENPA & 35.8 & 38.1 & 27.9 & $6.44 * *$ \\
\hline 11. Desesperación & ENDFS & 34.1 & 37.1 & 23.5 & $11.77 * * *$ \\
\hline 12. Aburrimiento & ENDFS & 33.4 & 33.1 & 34.4 & 0.11 \\
\hline 13. Irritabilidad & ENDFS & 33.1 & 35.7 & 24 & $8.76 * *$ \\
\hline 14. Tristeza & ENDFS & 32.9 & 36.5 & 20.2 & $17.10 * * *$ \\
\hline 15. Desmotivación & ENDFS & 30.7 & 33.2 & 21.9 & $8.65^{* *}$ \\
\hline 16. Insatisfacción & ENDFS & 29.9 & 30.7 & 26.8 & 1.06 \\
\hline 17. Desgano & ENDFS & 29.5 & 32.6 & 18.6 & $13.46 * * *$ \\
\hline 18. Fragilidad & ENPA & 28.6 & 30.4 & 22.4 & $4.47^{*}$ \\
\hline 19. Desconfianza & ENPEB & 24.4 & 25.3 & 21.3 & 1.21 \\
\hline 20. Soledad & ENDFS & 24 & 24.5 & 22.4 & 0.34 \\
\hline 21. Desaliento & ENPEB & 22.8 & 25.6 & 13.1 & $12.57 * * *$ \\
\hline 22. Apatía & ENDFS & 22.2 & 21.1 & 26.2 & 2.20 \\
\hline 23. Pesimismo & ENDFS & 20.5 & 21.5 & 16.9 & 1.83 \\
\hline 24. Hastío & ENDFS & 20.3 & 19.3 & 23.5 & 1.51 \\
\hline 25. Amargura & ENDFS & 20.3 & 21.2 & 16.9 & 1.61 \\
\hline 26. Desesperanza & ENPEB & 17.5 & 19.8 & 9.3 & $10.93 * *$ \\
\hline 27. Descontrol & ENPEB & 16.7 & 17.8 & 13.1 & 2.22 \\
\hline 28. Desasosiego & ENPEB & 14.8 & 16.1 & 10.4 & 3.64 \\
\hline 29. Pánico & ENPPI & 14.3 & 16.5 & 6.6 & $11.55^{* * *}$ \\
\hline 30. Duelo & ENPEB & 14 & 15.9 & 7.1 & $9.19 * *$ \\
\hline 31. Desconsuelo & ENPEB & 13.6 & 15.6 & 6.6 & $9.9 * *$ \\
\hline 32. Desamparo & ENPEB & 13.5 & 14.8 & 8.7 & $4.51 *$ \\
\hline 33. Terror & ENPPI & 11.3 & 12.3 & 7.7 & 3.10 \\
\hline 34. Desdicha & ENPEB & 10.4 & 11.5 & 6.6 & 3.78 \\
\hline 35. Pavor & ENPPI & 10.3 & 11.7 & 5.5 & $5.98 * *$ \\
\hline 36. Recelo & ENPEB & 9.7 & 10.0 & 8.7 & 0.25 \\
\hline
\end{tabular}

Nota: En la tabla de prevalencia se incluyeron a personas que respondieron que la emoción estaba "más o menos presente", "muy presente" o "demasiado presente". En consecuencia, quedaron excluidas las respuestas "nada presente" y "poco presente". Las emociones están ordenadas según su nivel de frecuencia en la muestra total.

ENDFS = Emociones Negativas por Distanciamiento Físico y/o Social.

ENPEB = Emociones Negativas por Pérdida/ausencia de Estabilidad o Bienestar.

ENPA $=$ Emociones Negativas por Percepción de Amenaza .

ENPPI = Emociones Negativas por Percepción de Peligro Intenso.

$* p<0.05, * * p<0.01, * * * p<0.001$ 
Como podrá apreciarse en la Tabla 1, los porcentajes más elevados de las 36 emociones negativas de este estudio se ubicaron principalmente en las dimensiones ENPA y ENDFS y, en menor medida, en las ENPEB y ENPPI. Al desagregar las medias para cada una de las cuatro dimensiones del inventario, se encontró que la del valor más alto fue aquella que correspondía a las "Emociones negativas por percepción de amenaza" (ENPA) ( $\bar{x}=2.5973$; $D E=0.90263)$; seguida por las "Emociones negativas por distanciamiento físico y/o social (ENDFS) $(\bar{x}=2.2042 ; D E=0.84280)$; las "Emociones negativas por pérdida/ausencia de estabilidad o bienestar (ENPEB) $(\bar{x}=1.7421$; $\mathrm{DE}=0.72684)$; y las "Emociones negativas por percepción de peligro intenso" (ENPPI) $(\overline{\mathrm{x}}=1.5777$; $\mathrm{DE}=0.88301)$.

Desagregando las emociones más experimentadas por grupo etario, se encontró que los jóvenes (18 a 24 años de edad) experimentaron incertidumbre $(66.6 \%, n=197)$, flojera $(65.2 \%, n=193)$, impaciencia $(59.5 \%, \mathrm{n}=176)$ y fastidio $(58.1 \%, \mathrm{n}=172)$. Por su parte, las emociones más experimentadas por el grupo de "adultos jóvenes" (25 a 44 años de edad) fueron incertidumbre $(62 \%, n=212)$, preocupación (53.8\%, $n=184)$, impotencia $(48.2 \%, n=165)$ e impaciencia $(45.6 \%, n=156)$. Los "adultos maduros" (45 a 59 años) experimentaron mayor sensación de incertidumbre (44.2\%, $n=65)$, preocupación (36.1\%, $n=53)$, impotencia $(34.7 \%, n=51$ ) y vulnerabilidad (27.2\%, n=40). Finalmente, los "adultos mayores" (60 años o más) reportaron mayor preocupación $(43.6 \%, n=17)$, sensación de impotencia $(33.3 \%, n=13)$, vulnerabilidad $(33.3 \%, n=13)$ e inseguridad $(25.6 \%, n=10)$.

Por su parte, utilizando un punto de corte de 17 (Villa, et al., 2013), se identificó que 31.6\% de la muestra de este estudio presentó malestar psicológico. La media aritmética de las mujeres ascendió a $14.28(\mathrm{DE}=6.763)$ y la de los hombres a $11.86(\mathrm{DE}=6.319)$.

Como puede apreciarse en la Tabla 2, sentirse incapaz de disfrutar actividades (53.6\%), problemas de concentración (51.9\%), sentirse agobiado (46.6\%), deprimido (37.6\%) y la pérdida de sueño (35.4\%), fueron los cinco principales malestares reconocidos por los propios participantes. Por otra parte, los malestares menos presentes en esta muestra fueron la sensación de no valer nada $(11.7 \%)$, la pérdida de confianza $(18.1 \%)$ y sentirse incapaz de hacer frente a los problemas (20.4\%). El resto de los porcentajes reportados pueden verse en la Tabla 2.

Tabla 2. Porcentajes obtenidos en cada reactivo del Cuestionario General de Salud (CGS).

\begin{tabular}{lcc}
\hline \multicolumn{1}{c}{ Reactivo } & Sí* $\boldsymbol{n} / \%$ & No $\boldsymbol{n} / \%$ \\
\hline 1. Problemas de concentración. & $428(51.9)$ & $396(48.1)$ \\
2. Pérdida de sueño. & $292(35.4)$ & $532(64.6)$ \\
3. Pensar que no se juega un papel útil en la vida. & $264(32.0)$ & $560(68.0)$ \\
4. Sentirse incapaz de tomar decisiones. & $179(21.7)$ & $645(78.3)$ \\
5. Sentirse agobiado y con tensión. & $384(46.6)$ & $440(53.4)$ \\
6. No poder superar dificultades. & $225(27.3)$ & $599(72.7)$ \\
7. Sentirse incapaz de disfrutar actividades. & $442(53.6)$ & $382(46.4)$ \\
8. Sentirse incapaz de hacer frente a problemas. & $168(20.4)$ & $656(79.6)$ \\
9. Sentirse deprimido. & $310(37.6)$ & $514(62.4)$ \\
10. Pérdida de confianza. & $149(18.1)$ & $675(81.9)$ \\
11. Sensación de no valer nada. & $96(11.7)$ & $728(88.3)$ \\
12. Sentirse menos feliz que lo habitual. & $289(35.1)$ & $535(64.9)$ \\
\hline
\end{tabular}

Nota: *Las respuestas a los reactivos del Cuestionario General de Salud-12 tienen cuatro opciones $(0,1,2$ y 3$)$. Elegir las opciones dos y tres en cada reactivo implicó la presencia de ese malestar. Por ello, en la columna "Sí" de esta Tabla se muestran las frecuencias sumadas de las opciones 2 y 3, las cuales aluden la presencia de cada uno de dichos malestares. 
Debido a que la muestra no cumplió con los requisitos de las pruebas paramétricas, para evaluar las diferencias entre las variables se empleó la prueba $U$ de Mann-Whitney. Con respecto a los puntajes totales de las emociones negativas (ENG), en este estudio se identificó que las mujeres (Rango promedio $=433.03, n=641$ ) tuvieron puntajes significativamente más elevados que los hombres (Rango promedio $=340.57, n=183$ ), $U=45489, z=-4.63, p=0.001,1-\beta=0.96, d=0.38$. Al desagregar las diferencias por cada una de las cuatro dimensiones del IREN (ENDFS, ENPEB, ENPA, ENPPI) y del malestar psicológico, se encontró que en todas ellas las mujeres también se ubicaron por encima de los hombres, como puede apreciarse en la Tabla 3.

Tabla 3. Diferencias por género, grupo de edad y escolaridad en cada una de las dimensiones de Emociones Negativas y Malestar Psicológico.

\begin{tabular}{|c|c|c|c|c|c|c|c|}
\hline \multirow{2}{*}{ Variables } & \multicolumn{2}{|c|}{ Rango promedio } & \multirow{2}{*}{$\mathbf{Z}$} & \multirow{2}{*}{$\begin{array}{l}\text { U de } \\
\text { Mann }\end{array}$} & \multirow{2}{*}{$\mathbf{p}$} & \multirow{2}{*}{$1-\beta$} & \multirow{2}{*}{ d } \\
\hline & Mujeres $\mathrm{n}=641$ & Hombres $\mathrm{n}=183$ & & & & & \\
\hline ENG & 433.03 & 340.57 & -4.63 & 45489 & .001 & .96 & 0.38 \\
\hline ENDFS & 426.69 & 362.81 & -3.20 & 49558.5 & .001 & .74 & 0.27 \\
\hline ENPEB & 430.30 & 350.16 & -4.02 & 47244 & .001 & .90 & 0.33 \\
\hline ENPA & 437.35 & 325.44 & -5.61 & 42720 & .001 & .99 & 0.45 \\
\hline ENPPI & 429.83 & 351.78 & -4.17 & 47540 & .001 & .86 & 0.31 \\
\hline MP & 433.41 & 339.27 & -4.72 & 45250.5 & .001 & .95 & 0.36 \\
\hline Variables & $\begin{array}{c}\text { Jóvenes y adultos } \\
\text { jóvenes* } n=638\end{array}$ & $\begin{array}{c}\text { Adultos maduros } \mathbf{y} \text { adultos } \\
\text { mayores** } \mathrm{n}=186\end{array}$ & $\mathbf{z}$ & $\begin{array}{l}\text { Ude } \\
\text { Mann }\end{array}$ & $\mathbf{p}$ & $1-\boldsymbol{\beta}$ & d \\
\hline ENG & 448.44 & 289.24 & -8.028 & 36407 & .001 & .99 & 0.66 \\
\hline ENDFS & 458.41 & 255.03 & -10.259 & 30044 & .001 & 1 & 0.91 \\
\hline ENPEB & 427.12 & 362.34 & -3.274 & 50004 & .001 & .78 & 0.28 \\
\hline ENPA & 435.54 & 333.46 & -5.154 & 44632 & .001 & .99 & 0.42 \\
\hline ENPPI & 431.89 & 345.99 & -4.622 & 46964 & .001 & .92 & 0.34 \\
\hline MP & 446.02 & 297.52 & -7.497 & 37947 & .001 & .99 & 0.64 \\
\hline Variables & $\begin{array}{c}\text { Sin estudios } \\
\text { profesionales } n=91\end{array}$ & $\begin{array}{l}\text { Con estudios profesionales } * * * \\
\qquad=733\end{array}$ & $\mathbf{Z}$ & $\begin{array}{l}\text { U de } \\
\text { Mann }\end{array}$ & $\mathbf{p}$ & $1-\boldsymbol{\beta}$ & d \\
\hline ENG & 382.91 & 416.17 & -1.258 & 30658.5 & .208 & .36 & 0.09 \\
\hline ENDFS & 374.91 & 417.17 & -1.598 & 29930.5 & .110 & .53 & 0.19 \\
\hline ENPEB & 409.77 & 412.84 & -.116 & 33103 & .907 & .91 & 0.04 \\
\hline ENPA & 367.55 & 418.08 & -1.912 & 29261.5 & .056 & .45 & 0.20 \\
\hline ENPPI & 465.56 & 405.91 & -2.406 & 28523 & .016 & .40 & 0.24 \\
\hline MP & 384.62 & 415.96 & -1.186 & 30814 & .235 & .48 & 0.12 \\
\hline
\end{tabular}

Notas:

*El rango de edad del grupo de "jóvenes" y "adultos jóvenes" es de 18 a 44 años.

**El rango de edad de los "adultos maduros" y "adultos mayores" es de 45 en adelante.

$* * *$ Las personas que aquí aparecen en el grupo denominado "con estudios profesionales" incluyen a los estudiantes de licenciatura y posgrado y a quienes ya finalizaron su formación profesional.

ENG = Emociones Negativas Globales.

ENDFS = Emociones Negativas por Distanciamiento Físico y/o Social.

ENPEB = Emociones Negativas por Pérdida/ausencia de Estabilidad o Bienestar.

ENPA $=$ Emociones Negativas por Percepción de Amenaza.

ENPPI = Emociones Negativas por Percepción de Peligro Intenso.

MP = Malestar Psicológico.

Por su parte, al realizar la prueba $U$ de Mann-Whitney en la variable grupo de edad, también se encontraron diferencias significativas en todas ellas. En este caso particular, se decidió agrupar a las personas en dos conjuntos: por un lado, jóvenes y adultos jóvenes y, por el otro, adultos maduros y adultos mayores. En consecuencia, el primero grupo estuvo constituido por edades de 18 a 44 años y el segundo de 45 en adelante.

En todas las dimensiones de las emociones negativas, así como en el malestar psicológico se presentaron diferencias significativas (Tabla 3). Por ejemplo, en los puntajes globales de las 
emociones negativas (ENG), las personas de mayor edad - grupo de 45 años en adelante- (Rango promedio $=289.24, n=186$ ) se ubicaron por debajo de los más jóvenes, es decir, de aquellas personas de 18 a 44 años de edad (Rango promedio=448.44, $n=638$ ), $U=36407, z=-8.028$, $p=.001,1-\beta=.99, d=0.66$. De la misma forma, el grupo más joven (Rango promedio=446.02, $\mathrm{n}=638$ ) también presentó indicadores significativamente superiores de malestar psicológico que los de mayor edad (Rango promedio $=297.52, n=186$ ) , $U=37947, z=-7.497, p=.001,1-\beta=.99$, $\mathrm{d}=0.64$.

Con respecto a la escolaridad, no se encontraron diferencias significativas salvo en la dimensión ENPPI, como puede verse en la Tabla 3. En este factor, las personas con estudios superiores (Rango promedio $=405.91, \mathrm{n}=733$ ) presentaron puntajes significativamente más elevados que aquellos que no contaban con este tipo de estudios (Rango promedio=465.56, $\mathrm{n}=91$ ), $\mathrm{U}=28523$, $z=-2.406, p=.016,1-\beta=.40, d=0.24$.

\section{DISCUSIÓN}

Los principales resultados de la presente investigación revelan una alta presencia de emociones negativas y de malestar psicológico, durante las Fases 1 y 2 del Covid-19 en la ZMCM -es decir, la región geográfica que resultó más afectada por la pandemia en México.

En el caso específico de las emociones negativas, de los análisis estadísticos se desprende que existen emociones y sensaciones perturbadoras que son compartidas por un porcentaje elevado de personas, particularmente la incertidumbre, preocupación, fastidio, impotencia e inquietud. En promedio, dichas emociones se presentaron en cinco de cada diez personas de la muestra, lo que significa una prevalencia considerablemente alta.

Además, llama la atención que los porcentajes más elevados de estas emociones negativas y perturbadoras, se ubiquen principalmente en las dimensiones ENPA y ENDFS; es decir, en primera instancia, son aquellas emociones que surgen de la percepción objetiva y subjetiva de una amenaza a la integridad física y, en segundo lugar, aquellas que están estrechamente ligadas a los impactos psicológicos del distanciamiento físico y/o social por la pandemia.

En el caso de la dimensión ENPA, la percepción de una amenaza latente del Covid-19 -que incluye un posible contagio de uno mismo o de algún ser querido, así como su consecuencia más extrema, el fallecimiento por SARSCov-2- activa y desencadena un conjunto de emociones perturbadoras específicas como preocupación, incertidumbre, inquietud, vulnerabilidad y miedo, entre otras. Como es sabido, estas respuestas emocionales particulares pueden ser la antesala de problemas de ansiedad diversos, y por ello resulta importante su identificación temprana.

En el caso de la dimensión ENDFS, entre las emociones negativas más experimentadas por los participantes de este estudio y que podrían estar vinculadas con los efectos del distanciamiento físico y social propios de un contexto pandémico - como no salir de casa, tener contacto mínimo con personas externas al hogar, la realización de home-office, alejamiento de amigos y compañeros queridos, fricciones al interior de la familia nuclear, etc.- se encuentran principalmente el fastidio, la impaciencia, la desesperación, el aburrimiento y la irritabilidad, entre otras. En promedio tres de cada diez personas del estudio experimentaron justamente emociones clasificadas como ENDFS.

Por otra parte, al desagregar las emociones por grupo etario, se encontró que los jóvenes, los adultos jóvenes y los adultos maduros tuvieron a la "incertidumbre" como la sensación negativa más presente de todas, con un $66.6 \%, 62 \%$ y $44.2 \%$ respectivamente. En el caso de los adultos mayores, la "preocupación" fue la del porcentaje más alto (43.6\%), la cual fue compartida como segunda expresión emocional más recurrente por los adultos jóvenes y los adultos maduros. 
Los resultados anteriores muestran, en su conjunto, una relativa homogeneidad de respuestas en términos de ordenamiento emocional, es decir, de la presencia jerárquica de ciertas emociones independientemente del grupo etario al que se pertenezca. Además, pone énfasis en la importancia de dos de las respuestas emocionales predominantes en este estudio - es decir, incertidumbre y preocupación - las cuales, en caso de que persistan e intensifiquen su intensidad con el tiempo, pueden transformarse en algún tipo de trastorno de ansiedad. Como señalan Clark y Beck (2010), la ansiedad se fundamenta principalmente en el miedo, pero también en la preocupación y en la incertidumbre constante.

Con respecto a la presencia de malestar psicológico, en este estudio se encontró una prevalencia de $31.6 \%$, lo cual demuestra que casi tres de cada diez personas presentaron signos de malestar emocional en el contexto de la pandemia. Este elevado porcentaje puede representar un llamado de alerta que demande formular, implementar y/o fortalecer políticas públicas que coadyuven en la atención y afrontamiento de esta problemática, sobre todo cuando hay evidencia de que el malestar psicológico puede convertirse en algún tipo de trastorno (Espíndola, et al, 2006; Parrado-González, \& León Jariego, 2020; Villa, et al. 2013).

Los principales malestares asociados a la pandemia identificados en esta investigación se refieren a la incapacidad de disfrutar actividades, problemas de concentración, pérdida de sueño, y la presencia de síntomas depresivos. Este tipo de respuestas remiten no sólo a reacciones de tipo psicoafectivo, sino también a reacciones fisiológicas y de activación, como es el nerviosismo, las palpitaciones constantes, problemas estomacales o sudoración de manos (Clark, \& Beck, 2010; Espíndola, et al., 2006).

En suma, la presencia de emociones negativas y perturbadoras en las personas, así como la alta prevalencia de malestar psicológico, podrían ser la antesala de una problemática mayor que tendría que ser atendida en términos preventivos. Como se sabe, las emociones negativas son respuestas normales ante diversas situaciones de la vida; sin embargo, cuando estas son recurrentes y se extienden a lo largo del tiempo, resulta posible que se transformen en algún tipo de malestar mayor o, en casos extremos, en trastornos psiquiátricos graves (Flores-Morales. et al., 2021; Piqueras, et al., 2009; Piqueras, et al., 2010).

Con respecto a las diferencias por género, se encontró que las mujeres obtuvieron puntajes significativamente superiores en todas las variables (Tabla 3). Particularmente, en la dimensión ENPA se identificaron las diferencias más robustas; en este sentido, las mujeres fueron más sensibles a las amenazas del Covid-19 que los hombres. Lo anterior sugiere, en términos generales, que las mujeres se vieron emocionalmente más afectadas que los varones -0 , por lo menos, reconocieron abiertamente su impacto durante la pandemia.

Este conjunto de diferencias podría deberse más a la forma en que mujeres y hombres abordan, reconocen y manejan su esfera afectiva, que a indicadores reales de fortalezas o debilidades propios de cada género. Como señalan Lungu, et al. (2015), mientras las mujeres tienden a centrarse más en los sentimientos generados por los estímulos, los hombres son más pasivos hacia las emociones negativas, tratando de analizar y en ocasiones subestimar los estímulos y todos sus impactos.

En este sentido, los autores arriba citados enfatizan la existencia de al menos dos factores específicos -biológicos y culturales-, los cuales modulan la sensibilidad a las situaciones negativas asociadas a dichas emociones. Dicha sensibilidad aludida por Lungu et al. (2015), podría ser uno de los múltiples factores mediadores de las diferencias de género, y tendría uno de sus fundamentos en las diferencias sutiles del funcionamiento cerebral, particularmente en el sistema límbico. Esta mayor reactividad emocional de las mujeres también podría explicar entre otras cosas, la doble probabilidad de las mujeres de sufrir depresión y trastornos de ansiedad en comparación con los hombres. 
Y es que, ciertamente, las mujeres suelen presentar prevalencias más altas de ansiedad y depresión -incluso en algunos países es casi el doble (OMS, 2020b). En el caso particular de México, las mujeres experimentan más trastornos de ansiedad y del estado de ánimo que los varones (Cerecero-García, et al., 2020; Schnaas, 2017). En este sentido, las emociones negativas y el conjunto de signos propios del malestar psicológico, pueden ser elementos constitutivos, aunque no limitativos, de dichos trastornos (Flores-Morales, et al., 2021).

Con respecto a las diferencias por edad, se encontró que el grupo de jóvenes y adultos jóvenes experimentaron más emociones negativas y malestar psicológico que los adultos maduros y los adultos mayores. En este sentido, la tendencia encontrada fue que, a menor edad, mayor fue la presencia de emociones negativas y de malestar psicológico. Estas diferencias se ven particularmente claras en la dimensión ENDFS, la cual engloba a las emociones que surgen como resultado del distanciamiento físico entre las personas. La potencia estadística entre los dos grupos etarios fue bastante elevada $(1-\beta=1)$, así como el tamaño del efecto $(d=0.91)$. Todo esto muestra, en suma, que las experiencias de las personas durante el confinamiento por el Covid-19 en la ZMCM -algunas más estrictas y rígidas que otras - terminó por afectar principalmente a los más jóvenes.

\section{Como señalan Flores-Morales et al., (2021, p.16):}

En un contexto cultural como el de México, en donde la población está habituada a la interacción física y afectiva con el prójimo, la imposibilidad de salir de casa (por la pandemia) representó un duro golpe a su cotidianidad. La dificultad para convivir con amigos y familiares externos por el riesgo latente a la transmisión les exigió adaptarse a nuevas formas y dinámicas de relación e integración - regularmente de tipo virtual- las cuales no siempre fueron asimiladas de forma adaptativa.

En menor medida, pero aun con indicadores robustos, la diferencia entre ambos grupos de edad también se manifestó en la variable de malestar psicológico $(1-\beta=.99 ; d=0.66)$. Todos estos indicadores parecen señalar que una mayor edad representa un factor de protección psicoemocional en el contexto pandémico, pese a que los adultos maduros y los adultos mayores son justamente los grupos más vulnerables al virus SARS-Cov-2. En este orden de ideas, identificar algunas variables adicionales que pudieran dar cuenta de por qué las personas mayores han asimilado, regulado o gestionado de manera más adaptativa las emociones negativas - pese a ser un grupo físicamente vulnerable- representa uno de los múltiples retos para futuras investigaciones.

Con respecto a la escolaridad, se pudo observar que los impactos emocionales de la pandemia de Covid-19 en la ZMCM fueron homogéneos, puesto que los niveles de estudio no representaron algún tipo de protección psicoemocional para las personas. No obstante, la diferencia entre el número de participantes de cada submuestra pudo haber afectado los resultados que aquí se presentan, por lo que resultaría importante equilibrar su participación en futuros estudios.

\section{Limitaciones del Estudio}

Es conveniente señalar que entre las limitaciones identificadas en este estudio podrían figurar la exclusión de otras zonas geográficas de México que, igualmente, estuvieron afectadas de manera significativa por el virus SARS-Cov-2. En este sentido, resultaría importante diseñar una investigación más incluyente, en términos geográficos, y con un mayor número de variables independientes, que pudieran dar luz sobre la problemática de los impactos psicoemocionales de la pandemia. Asimismo, consideramos importante equilibrar más las muestras en futuras 
investigaciones, ya que un sector significativo de los participantes del presente estudio tenía o estaba cursando una carrera universitaria.

\section{CONCLUSIONES}

A partir de los resultados de la presente investigación -a saber, una elevada prevalencia de emociones negativas y de malestar psicológico en adultos de la Ciudad de México bajo el contexto de la pandemia de Covid-19-, es posible concluir de manera preliminar que ambos constructos son de suma importancia, ya que podrían tener efectos nocivos en la salud mental de las personas, particularmente porque están asociados con la generación y formación de diversos trastornos, ya sean de ansiedad o del estado de ánimo. Si no son atendidos de manera correcta y asertiva a través de políticas públicas, sanitarias e institucionales eficaces, dichas respuestas podrían representar un problema de salud pública de grave riesgo en un futuro a corto y/o mediano plazo.

\section{Financiamiento}

Esta investigación se realizó gracias al apoyo del Consejo Nacional de Ciencia y Tecnología (CONACYT) de México a través del Sistema Nacional de Investigadores (SNI) y de la propia Universidad Nacional Autónoma de México (UNAM).

\section{REFERENCIAS}

Calhoun. Ch., \& Solomon, R. (1984). ¿Qué es una emoción? Lecturas clásicas de psicología filosófica. Ciudad de México: FCE.

Cao, W., Fang, Z., Hou, G., Han, M., Xu, X., Dong, J., \& Zheng, J. (2020). The psychological impact of the COVID-19 epidemic on college students in China. Psychiatry Research, 112934. https://doi.org/10.1016/j.psychres.2020.112934.

Cerecero-García, M., Macías-González, F., Arámburo-Muro, T., \& Bautista-Arredondo, S. (2020). Síntomas depresivos y cobertura de diagnóstico y tratamiento de depresión en población mexicana. Salud Pública de México, 62(6), 840-850.

Chóliz, M. (2005). Psicología de la emoción. El proceso emocional. Madrid: Versión en línea. https://www.uv.es/choliz/Proceso\%20emocional.pdf

Clark, D.A., \& Beck, A.T. (2010). Terapia cognitiva para trastornos de ansiedad. Bilbao: Desclée de Brouwer.

Espíndola, J.G., Morales-Carmona, F., Díaz, E., Pimentel, D., Meza, P., Henales, C., Carreño, J., \& Ibarra, A.C. (2006). Malestar psicológico: algunas de sus manifestaciones clínicas en la paciente gineco-obstétrica hospitalizada. Perinatol Reprod Hum, 20(4).

Flores-Morales, R. (2020, agosto). Duelo sin réquiem. Edición especial Proceso, 59. 
Flores-Morales et al - Covid-19: Prevalencia de Emociones Negativas y Malestar Psicológico en México

Flores-Morales, R., Reidl-Martínez, L., Adame-Rivas, A.K., \& Reyes-Pérez, V. (2021). Construcción y validación del Inventario de respuestas emocionales negativas y perturbadoras en contextos de pandemia (IREN-35) en población mexicana. Nova Scientia, 13, Special Issue: COVID-19, 1-30. doi: https://doi.org/10.21640/ns.v13ie.2818

Gamonal, R. Montero, E., Matías, J., \& Roncero, C. (2020). Anxiety, worry and perceived stress in the world due to the COVID-19 pandemic, March 2020. Preliminary results. MedRxiv, Preprint. doi: https://doi.org/10.1101/2020.04.03.20043992

Gao, J., Zheng, P., Jia, Y., Chen, H., Mao, Y., Chen, S.,... \& Dai, J. (2020). Mental health problems and social media exposure during COVID-19 outbreak. Plos One, 15, e0231924. https://doi. org/10.1371/journal.pone.0231924.

García, C. (1999). Manual para la utilización del Cuestionario de Salud General de Goldberg. Adaptación cubana. Revista Cubana de Medicina General Integral, 15(1), 88-97.

Godoy-Izquierdo, D., Godoy, J., López-Torrecillas, F. \& Sánchez-Barrera, M. (2002). Propiedades psicométricas de la versión española del "Cuestionario de Salud General de Goldberg -28". Revista de Psicología de la Salud, 1 (14), 49-71.

Goldberg, D.P., \& Hillier, V.F. (1979). A scaled version of the General Health Questionnaire. Psychological Medicine, 9, 139-145.

Goldberg, D., \& Williams, P. (1988). A user's guide to the General Health Questionnaire. Windsor, UK: NFER-Nelson.

González Ramírez LP, Martínez Arriaga RJ, Hernández-Gonzalez MA, \& De la Roca-Chiapas JM, (2020). Psychological Distress and Signs of Post-Traumatic Stress in Response to the COVID-19 Health Emergency in a Mexican Sample. Psychology Research and Behavior Management, 13, 589-597. https://doi.org/10.2147/PRBM.S259563

Hernández, R., Fernández, C. \& Baptista, P. (2014). Muestreo en la investigación cualitativa. En Metodología de la investigación. México: McGrawHill.

Le Breton, D. (2013). Por una antropología de las emociones. Revista Latinoamericana de Estudios sobre Cuerpos, Emociones y Sociedad. 10 (4), 69-79. http://www.relaces.com.ar/index. php/relaces/article/viewFile/208/145

Liu, N., Zhang, F., Wei, C., Jia, Y., Shang, Z., Sun, L., ... \& Liu, W. (2020). Prevalence and predictors of PTSS during COVID-19 outbreak in China hardest-hit areas: Gender differences matter. Psychiatry Research, 112921. doi: https://doi.org/10.1016/j.psychres.2020.112921.

Lungu, O., Potvin, S., Tikàsz, A., \& Mendrek, A. (2015). Sex differences in effective fronto-limbic connectivity during negative emotion processing. Psychoneuroendocrinology, 62, 180-188. doi: https://doi.org/10.1016/j.psyneuen.2015.08.012.

Marina, J.A. (1999). El laberinto sentimental. Barcelona: Compactos Anagrama.

Organización Mundial de la Salud. (2020a). Fase 3 de la pandemia COVID-19 en México. OMS advierte que ningún país está totalmente preparado. Obtenido el 23 de abril de 2021. https:// coronavirus.onu.org.mx/Fase-3-de-la-pandemia-covid-19-en-mexico-oms-advierte-queningun-pais-esta-totalmente-preparado-dia-de-la-tierra-en-peligro-escucha-las-noticiasde-la-onu 
Organización Mundial de la Salud. (30 de enero 2020b). Depresión. Obtenido el 27 de abril de 2021. https://www.who.int/es/news-room/fact-sheets/detail/depression

Organización Mundial de la Salud. (2021). COVID-19. Weekly Epidemiological Update. Obtenido el 25 de noviembre de 2021. file:///C:/Users/Rogelio/Downloads/20211109_Weekly_Epi_ Update_65.pdf

Ortony, A., Clore, G. \& Collins, A. (1996). La estructura cognitiva de las emociones. Siglo XXI.

Parrado-González, A. \& León-Jariego, J. (2020). Covid-19: factores asociados al malestar emocional y morbilidad psíquica en población española. Revista Española de Salud Pública. 94, 1-16.

Piqueras, J., Ramos, V., Martínez, A., \& Oblitas, L. (2009). Emociones negativas y su impacto en la salud mental y física. Suma Psicológica, 16(2), 85-112.

Piqueras, J., Ramos, V., Martínez, A. y Oblitas, L. (2010). Emociones Negativas y Salud. Universidad Femenina del Sagrado Corazón, 18 (1), 33-57.

Priego-Parra, B., Triana-Romero, A., Pinto-Gálvez, S., Durán, C., Salas-Nolasco, O., Manríquez, M., Ramos-de-la-Medina, A., \& Remes-Troche, J. M. (2020). Anxiety, depression, attitudes, and internet addiction during the initial phase of the 2019 coronavirus disease (COVID-19) epidemic: A cross-sectional study in México. MedRxiv. https://doi.org/10.1101/2020.05.10.20095844

Redacción AN. (abril 21, 2020). Declara México inicio de la Fase 3 del coronavirus. 23 abril de 2020, de Aristegui Noticias Sitio web: https://aristeguinoticias.com/2104/mexico/declaramexico-inicio-de-la-Fase-3-del-coronavirus/

Rodríguez, M., Jones, G. M., \& Benedicto, M.G. (2021). Covid-19: Ansiedad de rasgo y estado en una población seleccionada de Argentina en un contexto de cuarentena extendida. Revista Argentina de Salud Pública. Suplemento Covid-19, 13, 1-10.

Roy, D., Tripathy, S., Kumar, S., Sharma, N., Kumar, S., \& Kaushal, V. (2020). Study of knowledge, attitude, anxiety \& perceived mental healthcare need in Indian population during COVID-19 pandemic. Asian Journal of Psychiatry, 51, 1-7. doi: https://doi.org/10.1016/j. ajp. 2020.102083

Sandín, B., Valiente, R. M., García-Escalera, J., \& Chorot, P. (2020). Impacto psicológico de la pandemia de COVID-19: Efectos negativos y positivos en población española asociados al periodo de confinamiento nacional. Revista de Psicopatología y Psicología Clínica, 25(1), 1-22. https://doi.org/10.5944/rppc.27569

Schnaas, F. (2017). Avances en el diagnóstico y tratamiento de la ansiedad y la depresión. Neurología, Neurocirugía y Psiquiatría, 45 (1): 22-25.

Secretaría de Salud. (2020). Comunicado Técnico Diario Covid-19 México, 30 junio de 2020. Secretaría de Salud.

Shaughnessy, J. J., Zechmeister, E. B., \& Zechmeister, J. S. (2007). Métodos de investigación en psicología. México: McGrawHill 
Solís-Cámara, P., Meda, R., Moreno, B., \& Juárez, P. (2016). Estructura factorial del Cuestionario de Salud General GHQ-12 en población general de México. Salud \& Sociedad, 7, 62-76. DOI: 10.22199/S07187475.2016.0001.00004

Wang, C., Pan, R., Wan, X., Tan, Y., Xu, L., McIntyre, R...\& Ho, C. (2020). A longitudinal study on the mental of general population during the Covid-19 epidemic in China. Brain, Behavior and Immunity, Article in press. doi: https://doi.org/10.1016/j.bbi.2020.04.028

Villa, I., Zuluaga, C., \& Restrepo, L.F. (2013). Propiedades psicométricas del Cuestionario de Salud General de Goldberg GHQ-12 en una institución hospitalaria de la ciudad de Medellín. Avances en Psicología Latinoamericana, 31(3), 532-545. 